\title{
DESTRUCTIVE D.C. BREAKDOWN IN “BUILT-UP” BARIUM STEARATE FILMS
}

\author{
V.K. AGARWAL $\dagger$ and V. K. SRIVASTAVA \\ Department of Physics, University of Roorkee, Roorkee, India
}

(Received October 4 1973; in final form February 25, 1974)

\begin{abstract}
The thickness dependence of maximum breakdown voltage (d.c.) leading to the destruction of the film has been reported for "built-up" barium stearate films in the thickness range ( $400 \AA-1000 \AA)$. These films were chosen because of their promising applications for making dielectric devices, and because their thickness is accurately known and controllable (within $25 \mathrm{~A}$ ). The breakdown voltage is found to be almost independent of thickness and microscopic observations in transmission did not show the occurrence of "single-hole" and "propagating" breakdowns, as observed by Klein et al. in evaporated film systems. The I-V characteristics in the "non-destructive" phase have also been studied and the observed maximum breakdown voltage has been distinguished from the"maximum voltage breakdown" observed by Klein et al.
\end{abstract}

\section{INTRODUCTION}

Studies of the dielectric properties of evaporated thin films have interested many workers in recent years, because of the potential usefulness of such films in the development of electronic devices. Insulating thin "built-up" films of barium stearate studied in the present work have also been shown ${ }^{1-4}$ to be useful for making such thin film devices. Here, we have reported the thickness dependence of maximum breakdown voltage (d.c.) which determines the ultimate dielectric strength of the films, and permissible limit of operating voltages, these being the most important factors from the device application point of view.

Film capacitors having the geometry A1-film-A1 have been studied in the thickness range (400 $\AA-1000 \AA)$, corresponding to $16-40$ layers of barium stearate. Such thickness-dependence studies may also prove to be useful in the breakdown theory which is not yet fully understood, and in determining the mechanism of breakdown conduction. Under the carefully controlled conditions described, the "builtup" films are ideally suited for such studies, and for making devices, because of their easy reproducibility, smoothness, high dielectric strength and accurately known and controllable thicknesses (to within $25 \AA$ ). Earlier, we $e^{5,6}$ have reported the thickness depen-

†Presently working at Instituto di Fisica, Università degli Studi, Parma, Italy. dence of onset breakdown voltage (d.c. and a.c.), corresponding to the initial abrupt rise of current, on these films and a few other similarly built-up films.

A great amount of work on the breakdown behaviour of thin evaporated film systems has already been done by many workers and has been well reviewed recently ${ }^{7-9}$. Particularly, the destructive phase of breakdown has been extensively studied in the pioneering works of Budenstein ${ }^{10-12}$, Klein ${ }^{13-16}$ and their co-workers, for determining the basic mechanism of breakdown conduction. However, the two groups differed greatly at certain points in their discussions of the breakdown conduction mechanism. In the present work, the maximum breakdown voltage leading to the destruction of the film over large areas, and the I-V characteristics in the "non-destructive" phase have been studied. In the light of the work of Klein and Budenstein, it has been concluded that the "maximum breakdown voltage observed here is not characteristic of the bulk, and differs from that of Klein.

\section{EXPERIMENTAL}

Barium stearate films having the desired number of layers were deposited onto aluminized highly smooth "Gold Seal" microglass slides using the BlodgettLangmuir ${ }^{17-18}$ technique. Since the smoothness of the substrate is of crucial importance for the uniformity of films, the slides were carefully selected so 
that they had no visible scratches. Their planeness and smoothness were studied by the standard method of matching cleaned slide surfaces with a master optical flat. On proper illumination, the formation of reasonably straight, equidistant, parallel and smooth fringes thus ensured that the slide surfaces were almost plane ${ }^{19}$. The smoothness of such selected microglass slides can give a great improvement over that of the usual optical flat. ${ }^{20}$ This is, for example, revealed by the absence of "wriggle" in the fringes of equal chromatic order in extensive measurements of the small film thicknesses by Srivastava and Verma ${ }^{21,22}$ on a four-layer barium stearate film (thickness about $100 \AA$ ). These workers made use of "Gold Seal" microslides and found that, in general, the slides do not have sharp projections or waviness. The uniformity of the film also improved with an increasing number of layers, as pointed out earlier. ${ }^{23}$ In addition, the reproducibility of the onset breakdown voltage at low thicknesses measured earlier on these films also shows their high degree of uniformity. 5,6

Small capacitors of areas ranging from 0.4 to $0.5 \mathrm{~cm}^{2}$ were obtained by sandwiching the barium stearate film between thermally evaporated A1 electrodes. It is now well established that these layers are stable under vacuum, and thermal evaporation does not cause any damage to the film. ${ }^{23}$ Subsequent measurements were made using $\mathrm{Hg}$ droplets to contact the Al electrodes at both ends of the sandwich. Direct contact by a solid probe was avoided to prevent disruption of the $\mathrm{Al}$ film near the contact. A 90-volt battery regulated by a linear wire-wound potentiometer $(\simeq 100 \mathrm{k})$ was used both with and without a standard series resistor $(\simeq 5 \mathrm{k})$ between the source and the film. The breakdown voltages corresponding to the onset of breakdown, destructive breakdown and maximum breakdown were studied with a precision VTVM. A large area of the capacitor was observed to have been destroyed at the maximum breakdown voltage. This can be avoided simply by removing the supply voltage within a few microseconds of breakdown. For further details of the deposition technique, sandwich fabrication and breakdown measurement method, reference should be made to our earlier paper. ${ }^{5}$

\section{RESULTS}

Figures 1 and 2 are typical graphical representations of the current density $(J)$ as a function of applied voltage $(V)$ in the non-destructive phase for a 40 -layer (thickness about $1000 \AA$ ) barium stearate sandwich having areas $0.5 \mathrm{~cm}^{2}$ and $0.4 \mathrm{~cm}^{2}$ respectively, at room temperature $\left(298^{\circ} \mathrm{K}\right)$, with and without the series resistor. Measurements of maximum breakdown voltage (d.c.) on barium stearate films have been carried out for the thickness range (400 $\AA-1000 \AA)$, corresponding to 16-40 layers.

The point (A) in the I-V characteristics of Figures 1 and 2 corresponds to the initial abrupt rise in

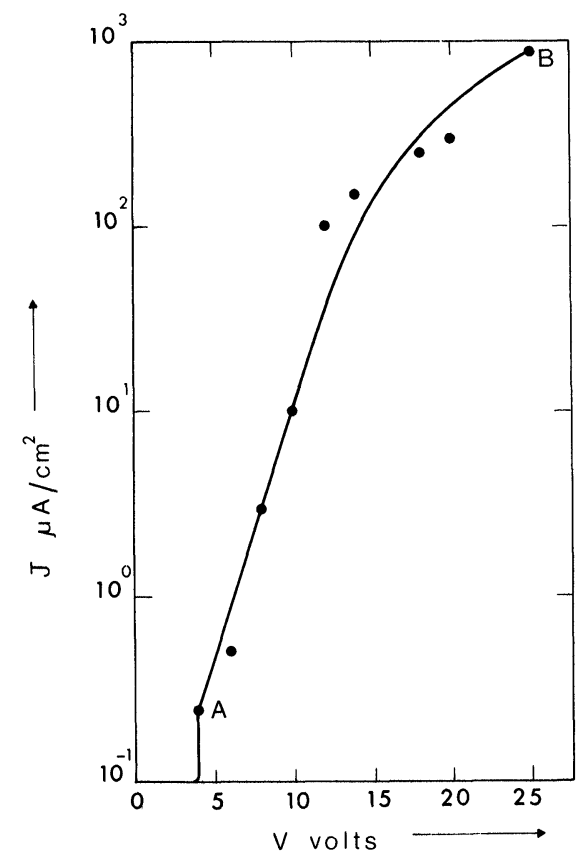

FIGURE 1 Plot of voltage $(V) \mathrm{v} / \mathrm{s}$ current density $(J)$ (with series resistor).

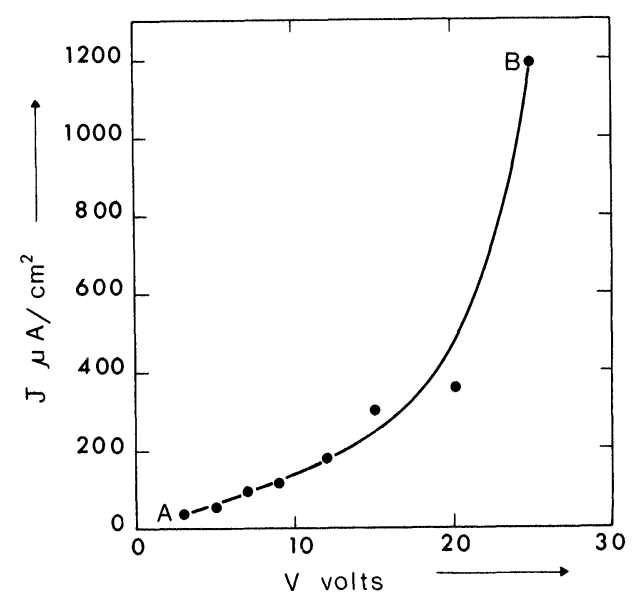

FIGURE 2 Plot of voltage $(V) \mathrm{v} / \mathrm{s}$ current density $(J)$ (without series resistor). 
current yielding the onset breakdown voltage, as has been studied in detail earlier. ${ }^{5}$ This breakdown voltage also agrees with the results shown in Figure 2 obtained without a series resistor. The point (B) in both Figures 1 and 2 corresponds to voltages where the "visible" destruction of the film commences. Beyond the point (B), the currents and voltages could not be properly recorded because of fluctuations, and therefore the I-V characteristics in the destructive phase have not been given here. For the films studied, the onset breakdown voltage ("A" in Figures 1 and 2) and the maximum breakdown voltage $\left(V_{\max }\right)$ are found to differ widely. Even the voltage at which the destruction commences (B) is considerably larger than the onset breakdown voltage (A).

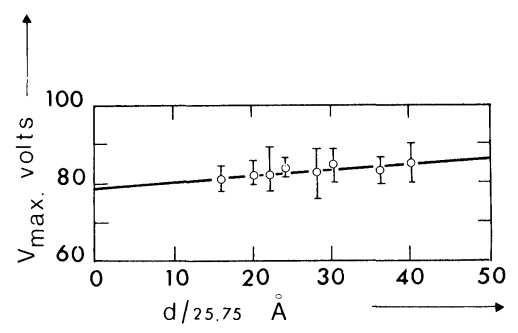

FIGURE 3 Plot of number of layers $(N)$ v/s maximum breakdown voltage $\left(V_{\max }\right)$.

Figure 3 shows graphically the thickness dependence of the maximum breakdown voltage $\left(V_{\max }\right)$. Figure 4 is a plot between the log of breakdown strength $\left(F_{\max }\right)$ and the $\log$ of film thickness $(d)$ (The value used for the thickness of a monolayer of barium stearate is $25.75 \AA .^{21}$ ) The maximum break down strength of barium stearate films for the range of thicknesses measured is $\simeq 10^{7} \mathrm{~V} / \mathrm{cm}$. The maximum breakdown voltage $\left(V_{\max }\right)$ is found to vary slightly with thickness and the corresponding breakdown

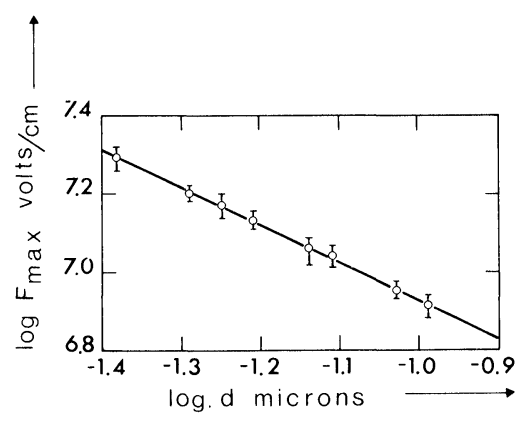

FIGURE 4 Plot of $\log$. of film thickness $(d) \mathrm{v} / \mathrm{s} \log$. of maximum breakdown strength $\left(F_{\max }\right)$.

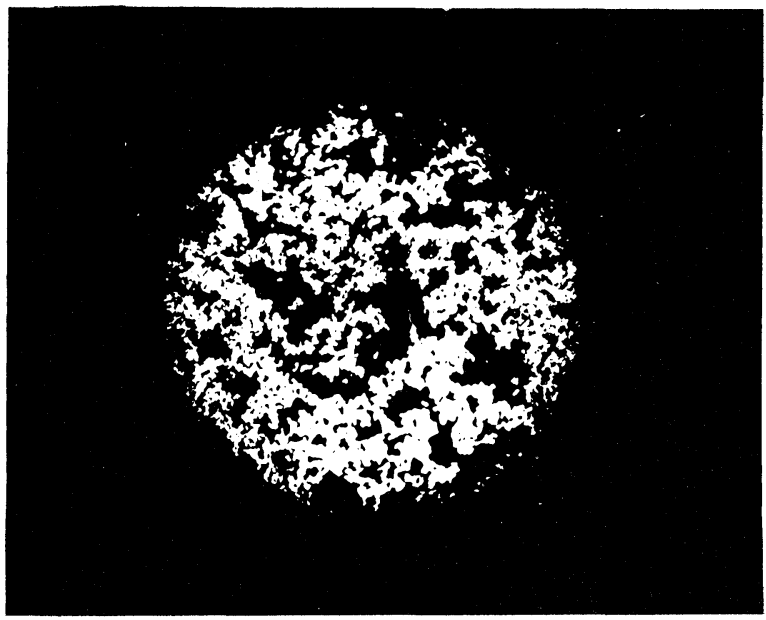

FIGURE 5 Transmission photomicrograph showing the destruction at maximum breakdown voltage $(x 40)$.

strength $\left(F_{\max }\right)$ is a power-dependent function of film thickness (d) varying as $d^{-\alpha}$ where $\alpha=0.96$.

Figure 5 is a representative transmission photomicrograph ( $x 40)$ showing the destruction of the film capacitor (area about $\left(0.4 \mathrm{~cm}^{2}\right)$, of thickness about $400 \AA$ (16 layers), over large areas at an applied voltage of 84 volts and with a series resistor $(\simeq 5 \mathrm{~K})$.

\section{DISCUSSION}

Klein and his co-workers ${ }^{13-16}$ classified destructive breakdown events in thin film dielectric as "single hole", "propagating" and "maximum voltage", and regarded the "maximum voltage breakdown" as characteristic of the ultimate dielectric strength of the bulk material. Budenstein and Hayes, ${ }^{10}$ however, showed that the maximum voltage breakdown" observed by Klein and Gafni $(\mathrm{K}-\mathrm{G})^{14}$ is initiated as a single-hole breakdown, and total destruction occurs through a series of consecutive breakdowns, each of these being localised and similar to other single-hole breakdowns. These authors ${ }^{10}$ thus showed that the classification of destructive breakdowns, referred to above, is pureiy phenomenological and the "maximum voltage breakdown" observed is not characteristic of the bulk dielectric. Thus, Budenstein and coworkers 10,11 did not encounter any such "maximum voltage breakdown".

The maximum breakdown voltage studied here is not characteristic of the bulk material and should be distinguished from the "maximum voltage breakdown" observed by K-G. ${ }^{14}$ Their data on "maximum 
voltage breakdown" was definitely based on the appearance of the bendover in the $\mathrm{I}-\mathrm{V}$ characteristic which they assumed to be an indication of impending destructive breakdown, although Budenstein and Hayes later pointed out that the bendover is essentially independent of the occurrence of destructive breakdown (in capacitors of large areas) which can be attributed to regions where there is considerable heating. The "maximum voltage breakdown" data of $\mathrm{K}-\mathrm{G}$ are thus based on the bendover, rather than on the voltage at which destruction occurs. Since they $(\mathrm{K}-\mathrm{G})$ were interested in the temperature-dependent studies of the breakdown on a single capacitor, they avoided going beyond the turnover voltage, which according to them was close to destruction voltage. Since we were not interested in the temperature effects on breakdown, the I-V characteristics have been plotted right up to the occurrence of "visible" destruction, and the onset breakdown voltage $V_{\mathrm{b}}$ (in Figures 1 and 2) is found to be widely different from the maximum breakdown voltage $V_{\max }$. In the present studies, no such bendover as observed by $\mathrm{K}-\mathrm{G}$ has been found in the $\mathrm{I}-\mathrm{V}$ characteristics, even when the series resistance was used. As the whole area is not destroyed in our case even at this maximum breakdown voltage (Figure 5), it differs basically from that of $\mathrm{K}-\mathrm{G}$ and determines the "ultimate dielectric strength" of the dielectric for all practical purposes from the device application point of view, but is not a characteristic of the bulk, as stated by $\mathrm{K}-\mathrm{G}$.

Microscopic observations in transmission did not show the occurrence of "single-hole" and "propagating" breakdowns of the type Klein et al. have observed in their work, because of the self-healing, non-shorting nature of the breakdown events and, presumably, because the evaporation hole was produced in only one of the thin electrodes. Their absence may also be attributed to the fact that organic films of barium stearate down to monolayer thickness are known to have high structural perfection and thus may be regarded as free from gross defects ${ }^{24}$ or "weak spots" unlike the films in evaporated systems. This factor seems to be further supported by Budenstein et al., who consider that the breakdown observed is not necessarily due to weak spots in the film.

Moreover, the basic difference between the present studies and those of Klein results from the nonthermal nature of our breakdown events. This has been justified by earlier measurements ${ }^{5}$ on the thickness dependence of the onset breakdown voltage in these film systems where the well-established electronic breakdown theory of Forlani and Minnaja ${ }^{7}$ is perfectly applicable. As the actual mechanism of destructive breakdown is not yet fully understood, no attempt has been made here to interpret the above results on 'destructive' breakdown. The interpretation of V-I characteristics in the "non-destructive" phase is also not possible as there is inadequate information about the conduction mechanism in the postbreakdown region. However, the data reported above may prove useful in device applications of the films.

\section{ACKNOWLEDGEMENT}

V. K. Agarwal acknowledges the financial assistance of the Council of Scientific and Industrial Research, New Delhi, India, for carrying out this work.

\section{REFERENCES}

1. J. L. Miles and H. O. McMahon, J. Appl. Phys. 32, 1176 (1961)

2. R. M. Handy and L. C. Scala, J. Electrochem. Soc. 113, 109 (1966)

3. P. L. Hawkes, Thin Film Integrated Circuits, Vol. 5, AEI Engineering, (1965) p. 304.

4. S. Horiuchi, J. Yamaguchi and K. Naito, J. Electrochem. Soc. 115, 634 (1968)

5. V. K. Agarwal and V. K. Srivastava, Thin Solid Films 8, 377 (1971); 13, S23 (1972)

6. V. K. Agarwal and V. K. Srivastava, J. Appl. Phys. 44, 2900 (1973)

7. F. Forlani and N. Minnaja, J. Vac. Sci. Technol. 6, 518 (1969)

8. N. Klein, Thin Solid Films 7, 149 (1971)

9. N. Klein, Advances Electron. Electron Phys. 26, 309 (1969)

10. P. P. Budenstein and P. J. Hayes, J. Appl. Phys. 38, 2837 (1967)

11. P. P. Budenstein, P. J. Hayes, J. L. Smith and W. B. Smith, J. Vac. Sci. Technol. 6, 289 (1969)

12. P. P. Budenstein and P. J. Hayes, J. Vac. Sci. Technol. 6 , 602 (1969)

13. N. Klein, H. Gafni and J. J. David, Proc. Symp. Phys. Failure Electron 3, 315 (1965)

14. N. Klein and H. Gafni, IEEE Trans. Electron Devices ED-13, 281 (1966)

15. N. Klein and Z. Lisak, Proc. IEEE 54, 979 (1966)

16. N. Klein and N. Levanon, J. Appl. Phys. 38, 3721 (1967)

17. K. B. Blodgett, J. Am. Chem. Soc. 57, 1007 (1935)

18. K. B. Blodgett and I. Langmuir, Phys. Rev. 51, 964 (1937)

19. J. Strong, Modern Physical Laboratory Practice (Blackie and Sons, London 1940)

20. S. Tolansky, An Introduction to Interferometry (Longmans Green, London 1955), p. 165

21. V. K. Srivastava and A. R. Verma, Proc. Phys. Soc. (London) 80, 222 (1962)

22. V. K. Srivastava and A. R. Verma, Solid State Commun 4, 367 (1966)

23. L. Holt, Nature 214, 1105 (1967)

24. K. L. Chopra, Thin Film Phenomena (McGraw-Hill, New York 1969), p. 406 

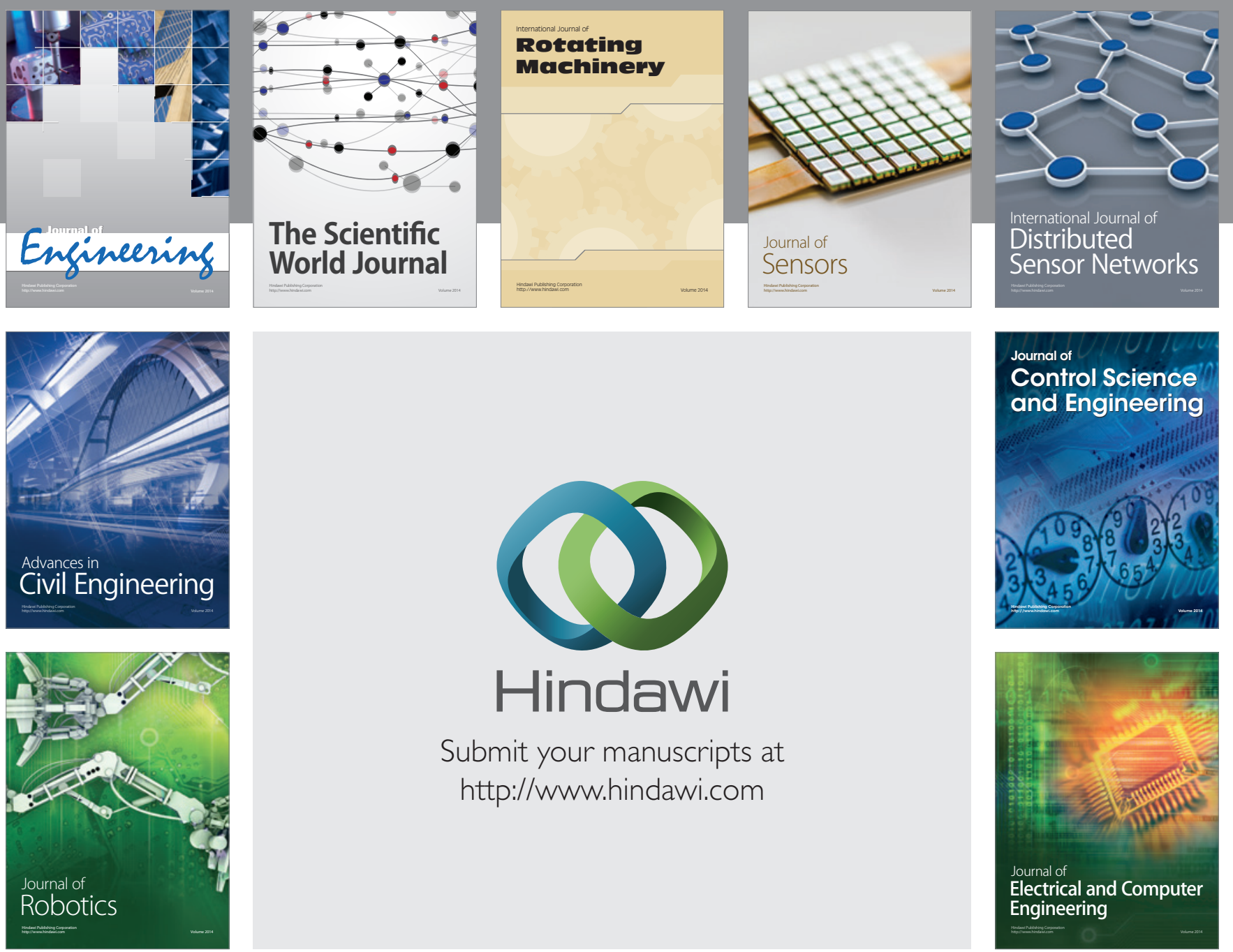

Submit your manuscripts at

http://www.hindawi.com
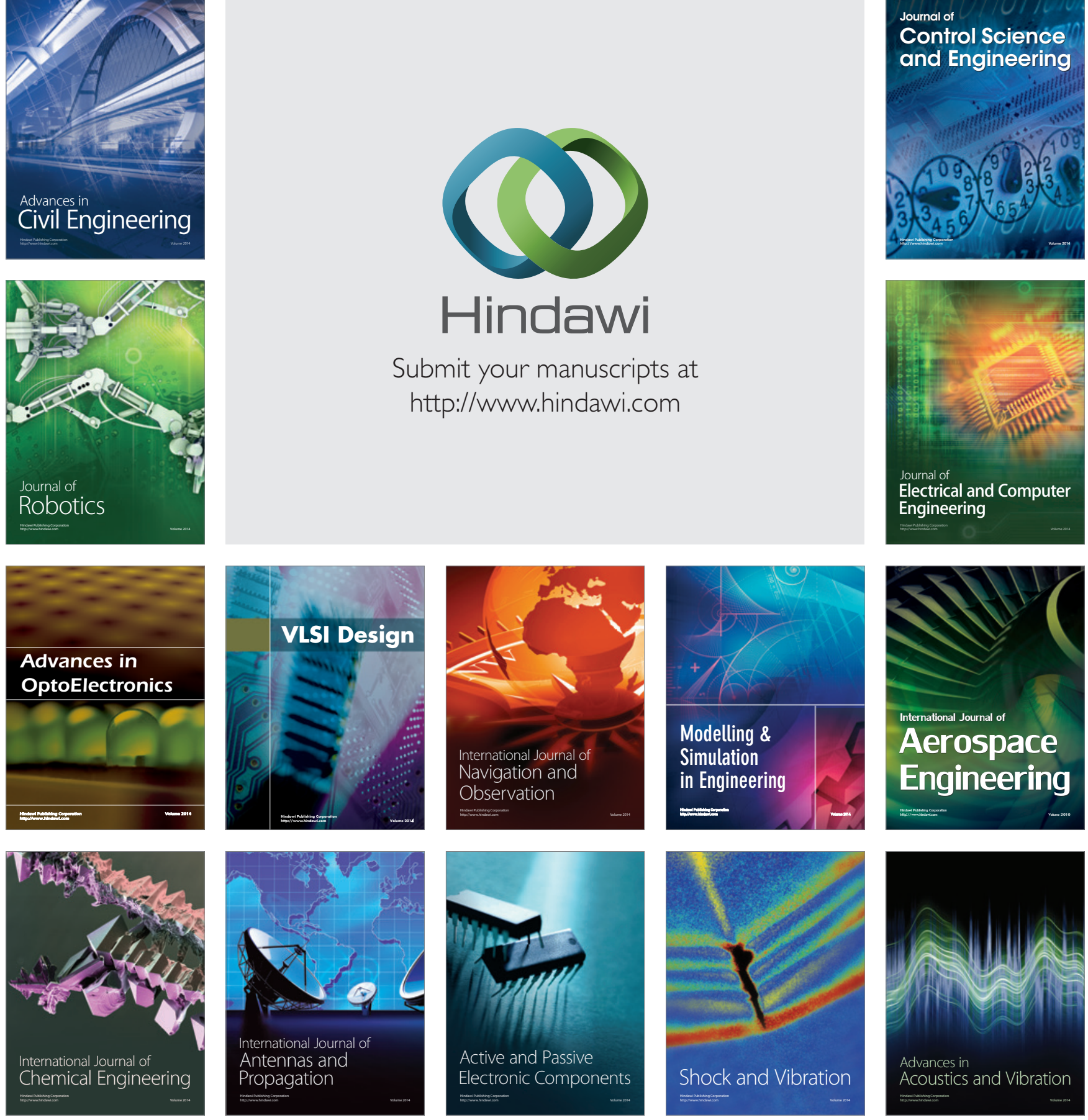